\title{
Susceptibility of a Shielded and an Unshielded Microstrip Transmission Line against a Near Interference Source
}

\author{
Sevda Abadpour ${ }^{1, *}$, Parisa Dehkhoda ${ }^{2}$, Ruzbeh Moini ${ }^{1}$, Seyyed Hesameddin Hossein Sadeghi ${ }^{1}$ \\ ${ }^{1}$ Electromagnetics Research Laboratory of Amirkabir University of Technology, 15914, Hafez ave., Tehran, Iran \\ ${ }^{2}$ Institute of Communications Technology and Applied Electromagnetics, Amirkabir University of Technology, 15914, \\ Hafez Ave., Tehran, Iran \\ * Corresponding Author: sevda.abadpour@aut.ac.ir
}

Copyright (C) 2014 Horizon Research Publishing All rights reserved.

\begin{abstract}
In this paper, the susceptibility of a microstrip transmission line (MTL) as a simple printed circuit board (PCB) near an interfering antenna is studied using the finite integral technique. Here, the magnitude of transmission coefficient of a two-port network model of the system $\left(\left|S_{21}\right|\right)$ is chosen as the susceptibility criterion of the PCB. The susceptibility is studied for the unshielded and shielded MTL. The effect of different perforation size on the susceptibility of the shielded MTL is examined and it is shown that large apertures can even increase the disagreeable effects of the interfering wave on the MTL at resonance bandwidth of the perforated enclosure. As an important result, it is shown that shielding effectiveness (SE) of an enclosure -which is defined based on plane wave incidence - can be used to predict the susceptibility behavior of the shielded MTL in the vicinity of interference. Simulation results are in good agreement with the measurements performed inside a semi-anechoic chamber.
\end{abstract}

Keywords Finite Integral Technique, Susceptibility Measurement, Shielding Enclosure, Transmission Line, Shielding Effectiveness

\section{Introduction}

Failure and malfunction of electronic systems that are sensitive to electromagnetic disturbances is becoming a serious problem [1]. The malfunctioning of an electronic system can be related to its susceptibility to an external electromagnetic field. As defined in [2], susceptibility is a relative measure of a device or a system's propensity to be disrupted or damaged by electromagnetic interference (EMI) exposure to an incident field.

The radiated susceptibility of a printed circuit board (PCB) influenced by an external electromagnetic field can be a criterion to determine the susceptibility of a whole electronic device. The induced interference signal on the traces of a PCB causes partial functional failures or even irreversible damages, depending on the shape and amplitude of the interference signal as well as the trace properties.

Generally speaking, metallic enclosures are widely used to hinder electromagnetic leakage from electronic equipments and also to reduce the susceptibility of the sensitive devices against external interference. However, in most practical applications, shielding effectiveness (SE), and consequently, the susceptibility of a PCB inside the enclosure are primarily affected by the apertures perforated to accommodate visibility, ventilation or access to interior components.

Numerous techniques have been utilized to evaluate the SE of perforated empty enclosures [3-8] and also loaded enclosures [9-13]. Also, various susceptibility measurements have been performed using a transverse electromagnetic transmission cell (TEM cell) [14], an asymmetric transverse electromagnetic transmission cell (ATEM cell) [15], gigahertz transverse electromagnetic transmission cell (GTEM cell) [3, 16] and reverberating chamber [17]. In those measurements, the induced voltage on different parts of PCB is usually defined as the susceptibility of the circuit.

In all mentioned studies, susceptibility of a circuit is investigated against an incident transverse electromagnetic (TEM) wave. Also, SE of an enclosure (that is the ratio of the field strength at one internal point in the presence and absence of the enclosure) is a definition based on plane wave interference. In this paper, the susceptibility of a microstrip transmission line (MTL) as a simple PCB near an interference source is studied. A simple MTL is selected to study, because in practical applications of microwave circuits, there exist various transmission lines like structures such as interconnecting wires and traces on printed circuit boards [18]. These transmission lines may play the role of a radiation exciter or receiver because of their antenna-like behavior. The effect of protecting the MTL against near interference by an enclosure with different apertures is also examined and it is shown that for large aperture cases at large frequency bandwidth, MTL immunity is degraded.

In addition, the effect of increasing the distance between the antenna and the MTL in the near field area is investigated and it is shown that small variations in distance do not affect 
the immunity of the MTL against a close interference. As an important result, it is shown that SE of the enclosure -which is a definition based on plane wave incidence- can predict the susceptibility of the shielded MTL. Hence, the size of the aperture or the location of the MTL inside the enclosure can be chosen by considering the SE of the considered enclosure at various points.

Here, the magnitude of the transmission loss $\left(\left|S_{21}\right|\right)$ between the emitting source (a monopole antenna) and the matched MTL is defined as the determinant parameter for susceptibility of the MTL [19]. Also, the voltage can be calculated from $\left|S_{21}\right|$, if needed. To evaluate $\left|S_{21}\right|$, two different tools are used here. The transmission loss is first calculated using the finite integral technique (FIT) with the well-known commercially available Computer Simulation Technology (CST) software [20] and then validated by measurements performed in a semi-anechoic chamber using a network analyzer.

The paper is organized as follows: in Section II, a brief description of theoretical model and the experimental set up is presented. The susceptibility of the MTL for different test configurations are discussed in Section III and concluded in Section IV.

\section{Problem Description}

Fig. 1 shows a schematic of a shielded MTL when exposed to the interfering wave of a close monopole antenna. A $6 \mathrm{~cm}$ monopole antenna is assumed to be the source of the interference and is designed for radiation at $1.25 \mathrm{GHz}$. The $80 \mathrm{~mm}$ microstrip transmission line is designed at $1 \mathrm{GHz}$ with substrate of RO4003. In cases where the enclosure is used, as shown in Fig. 1b, the MTL is placed inside the enclosure horizontally, in a way that the transmission line is parallel to the perforated wall and the antenna is placed outside of the enclosure as close as possible to the perforated wall. The vertical distance between the MTL and the antenna is $\mathrm{d}$ and is one of the considered variables in the problem. The enclosure dimension in all cases is $30 \times 12 \times 30 \mathrm{~cm}$ with a thickness of $\mathrm{t}=0.4 \mathrm{~cm}$.

The magnitude of transmission coefficient of a two-port network model of the system $\left(\left|S_{21}\right|\right)$ is defined as the susceptibility criterion of MTL, which is calculated numerically by CST and validated by measurements for some cases. The numerical simulation in CST is based on Maxwell's integral form equations that are applied in to the cells of a discretized problem space [21]. This approach is the basis of the finite integration technique (FIT) that is found to be a suitable numerical method for analyzing electromagnetic problems due to its high flexibility as well as its ability to deal with arbitrary material distributions and electrical properties. In the case of using a shielding enclosure, approximately 1,100,000 mesh cells are required to obtain a valid answer. The high number of meshes is due to the relatively small size of MTL when compared to the large size of the enclosure. On a Core ${ }^{\mathrm{TM}}$ i7-2600 CPU @ 3.4
$\mathrm{GHz}$, with $8 \mathrm{~GB}$ of RAM, the simulation time is about 250 sec.

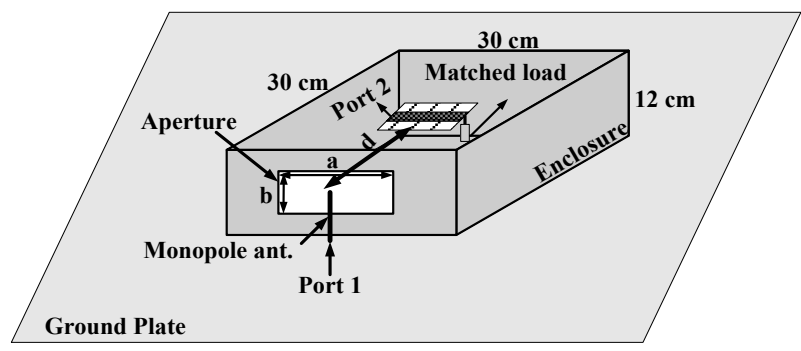

(a)

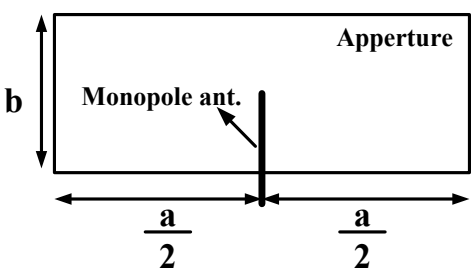

(b)

Figure 1. Schematic of an MTL inside an enclosure exposed to an interfering wave of a monopole antenna

Measurement is performed inside a semi-anechoic chamber using a Rohde \& Schwarz ZVK-40 GHz vector network analyzer (VNA). The first port of the VNA is connected to the monopole antenna and the second port is connected to the matched MTL. The fabricated enclosure has a replaceable perforated wall while the other walls are fully welded to each other to prevent field leakage from the edges. Fig. 2 shows the antenna location in the measurements of shielded MTL.

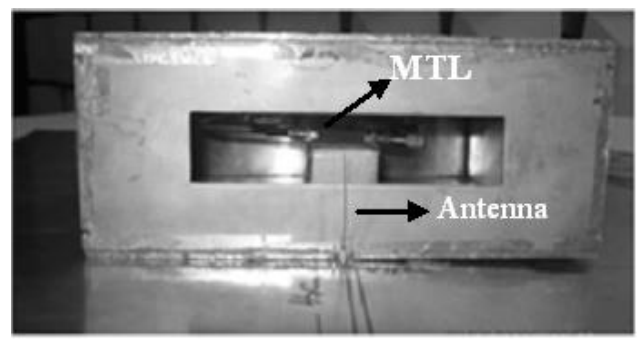

Figure 2. A picture of the fabricated MTL inside an enclosure when exposed to the radiation of a monopole antenna closely placed to the perforated wall.

\section{Results}

To study the susceptibility of an unshielded and a shielded MTL, we resort to CST simulation data and then conduct experimental tests for selected case studies. The effect of using shields with different aperture size along with the effect of distance between MTL and the antenna are studied. In addition, for the shielded cases, enclosure SE is depicted and it is shown that the susceptibility of a MTL against a near interference source can be predicted by SE of the enclosure 
which is a parameter defined based on a plane wave interference.

\subsection{Validation}

First, the obtained CST results for a matched MTL without a shield and also inside an enclosure with four apertures are validated with measurements. This step is necessary in order to be convinced about the essential number of cells in CST to get the accurate results. Furthermore, in the following sections whenever measurements are available they are compared with the simulation results.



(a)

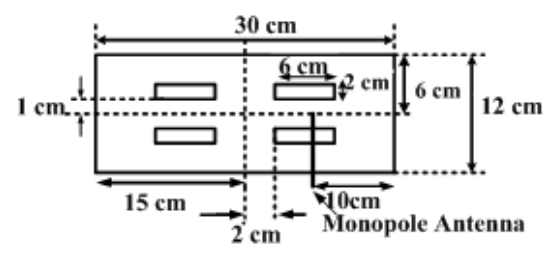

(b)

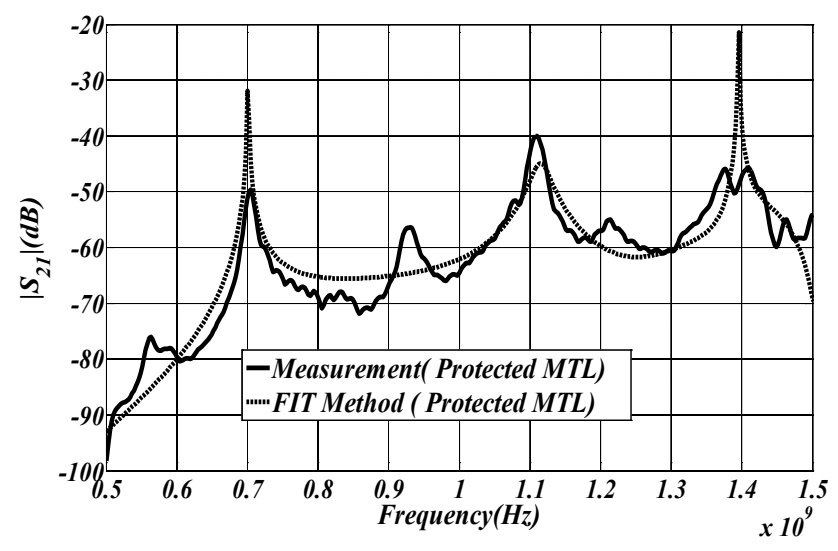

(c)

Figure 3. a) $\left|S_{21}\right|$ Comparison between CST and measurements when the shield is not used, b) Aperture configuration and location of the antenna, c) $\left|S_{21}\right|$ Comparison between CST and measurements when the shield is used

Fig. 3.a shows susceptibility $\left(\left|S_{21}\right|\right)$ of the unshielded matched MTL at $\mathrm{d}=15.4 \mathrm{~cm}$. Fig. 3.b shows the aperture configuration of a four-aperture enclosure and the location of the antenna. Shielded MTL is placed at the center of the enclosure as shown in Fig. 2 again with $\mathrm{d}=15.4 \mathrm{~cm}$. Fig 3.c shows susceptibility $\left(\left|S_{21}\right|\right)$ of the shielded matched MTL. A very good agreement is observed between results of CST and the measurements. Please note that, here, the Auto Regressive (AR) filter is applied in the simulations. Since AR filter predicts the signal for longer time rather than FIT simulation, it can eliminate the ripples [22].

Studied metallic cavity has the first nine resonant frequencies of $0.5,0.707,1,1.118,1.25,1.346,1.414,1.436$, 1.5 GHz. For the perforated enclosure, some resonance frequencies are cancelled due to symmetry and some other remain with a displacement according to the apertures size. If the electrical size of the apertures is small the resonance dip are not shifted very much in the frequency range. Vice versa, when the aperture size is large compared to the enclosure size, the $\mathrm{Q}$ factor of the enclosure decreases which leads to a wideband resonance. The effect of resonant frequencies can be detected also in $\left|S_{21}\right|$ plot of the system when MTL is placed inside the enclosure. In Fig. 3.c, $\left|S_{21}\right|$ has peaks at $0.7071,1.118$ and $1.4142 \mathrm{GHz}$ which are coincide with resonant frequencies of the box. Since the apertures are not large, the resonant frequencies are not shifted significantly. As observed, at mentioned frequencies, $\left|S_{21}\right|$ increases considerably and hence the immunity of MTL is decreased even compared to the case where the shield is not used.

It should be noted that due to aperture size, resonant frequencies of the apertures is not observed in the considered frequency range in the figures.

\subsection{Aperture Size}

The effect of various enclosures with different aperture sizes of $a^{\prime} \times \mathrm{b}(\mathrm{b}=5 \mathrm{~cm})$ and $a \times b^{\prime}(\mathrm{a}=18 \mathrm{~cm})$ on the susceptibility of the MTL are compared in Fig. 4.a and 4.b, respectively. In addition the result for the unshielded MTL is depicted in those figures. As in previous case, MTL is placed at $\mathrm{d}=15.4 \mathrm{~cm}$ and the antenna is places as close as possible to the centre of perforated wall as in Fig. 1.

The results confirm that decreasing the length of the aperture $\left(a^{\prime}\right)$ leads to reduce $\left|S_{21}\right|$ and hence improves the susceptibility of the MTL. However, at enclosure resonance frequencies, higher peaks with small bandwidth appear in $\left|S_{21}\right|$. Level of peaks is high compared to the unshielded MTL. The same result has been observed in Fig. 3. Hence, at resonance frequencies MTL can be affected highly by the incident wave. The results in Fig 4.b show that reduction of width $\left(b^{\prime}\right)$ does not change the $\left|S_{21}\right|$ significantly, while due to Fig 4.a, decreasing the $a^{\prime}$ improves $\left|S_{21}\right|$. This is because the incident electric field is normally polarized to the length of the aperture $\left(a^{\prime}\right)$.

For apertures with large lengths such as $a^{\prime}=18 \mathrm{~cm}$, $\left|S_{21}\right|$ gets higher values at a larger bandwidth compared to the case where no protection is used. This means that the shield acts conversely and does not protect MTL from the outside interference at a large bandwidth. To show the 
effect of aperture length statistically, Table 1 compares the average of MTL susceptibility for different aperture sizes over frequency. Average of susceptibility (AS) is calculated by (1).

$$
A S=\frac{\sum\left|S_{21}(f)\right| \times f}{\sum f}
$$

Since $\left|S_{21}\right|$ has high peaks at enclosure resonance frequencies, in calculation of $\mathrm{AS}$, a $100 \mathrm{MHz}$ bandwidth around each resonance $\left(f_{l}=704.8 \mathrm{MHz}, f_{2}=1113 \mathrm{MHz} \&\right.$ $\left.f_{3}=1420 \mathrm{MHz}\right)$ is excluded. Results in Table 1 reveal that aperture sizes of $4.5 \times 5 \mathrm{~cm}$ and $2.25 \times 5 \mathrm{~cm}$ increase the immunity of the MTL compared to the case where MTL is not protected by the enclosure. However, at a small bandwidth around resonance frequencies susceptibility of the MTL is increased.

The observed effect can be confirmed in Fig. 4c where $\mathrm{SE}$ of an empty enclosure with the same apertures and the same incident polarization is sketched. Illustrated SE is the ratio of the field strength in the presence and absence of the enclosure at the point inside the enclosure where MTL would be placed. In this definition the incident field is a plane wave. As observed in this figure, clearly, increasing the aperture size would increase the bandwidth where SE $(\mathrm{dB})$ is negative. This fact is due to decreasing the $\mathrm{Q}$ factor of the box for larger apertures and consequently, increasing the negative SE bandwidth. At this bandwidth the enclosure makes the outside interfering field resonate. It should be noted that although SE is defined for an incident plane wave, it can predict correctly the susceptibility of a shielded circuit against a near interference.

Table 1. Average of susceptibility for different aperture sizes

\begin{tabular}{|c|c|}
\hline Aperture size $\left(\mathrm{cm}^{2}\right)$ & AS (dB) \\
\hline $18 \times 5$ & -59.5629 \\
\hline $9 \times 5$ & -63.6208 \\
\hline $4.5 \times 5$ & -73.7481 \\
\hline $2.25 \times 5$ & -82.3320 \\
\hline Unprotected MTL & -63.1354 \\
\hline
\end{tabular}

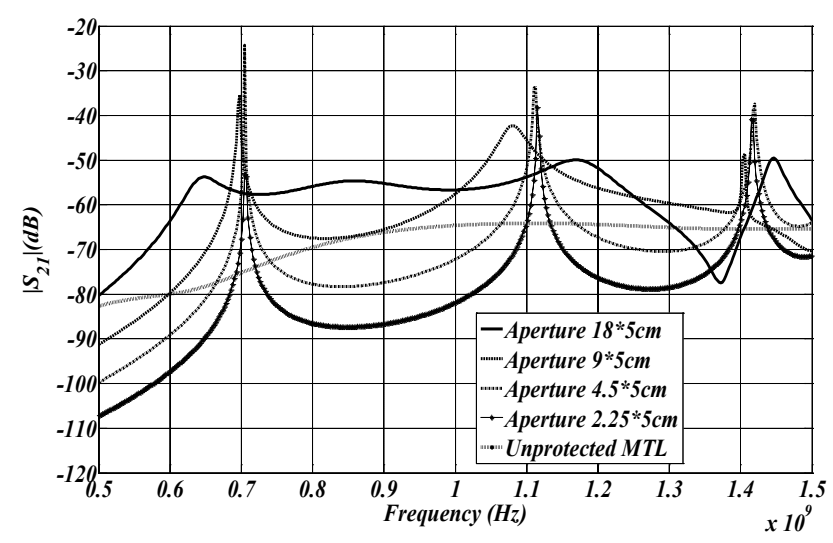

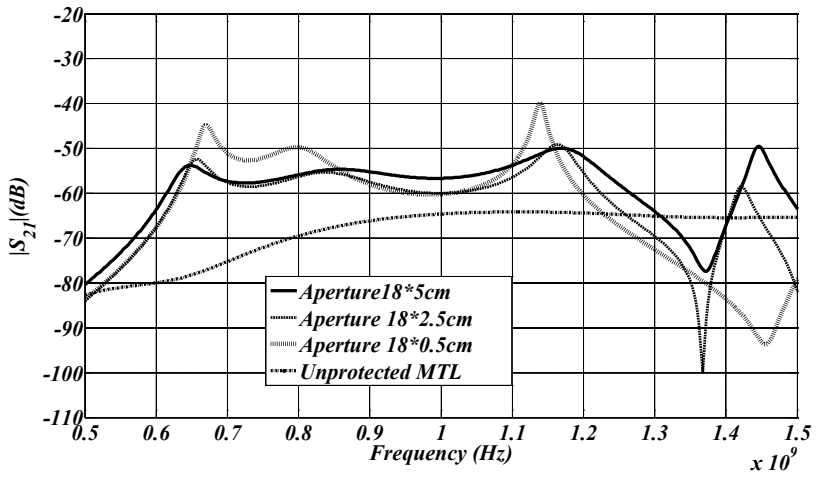

(b)

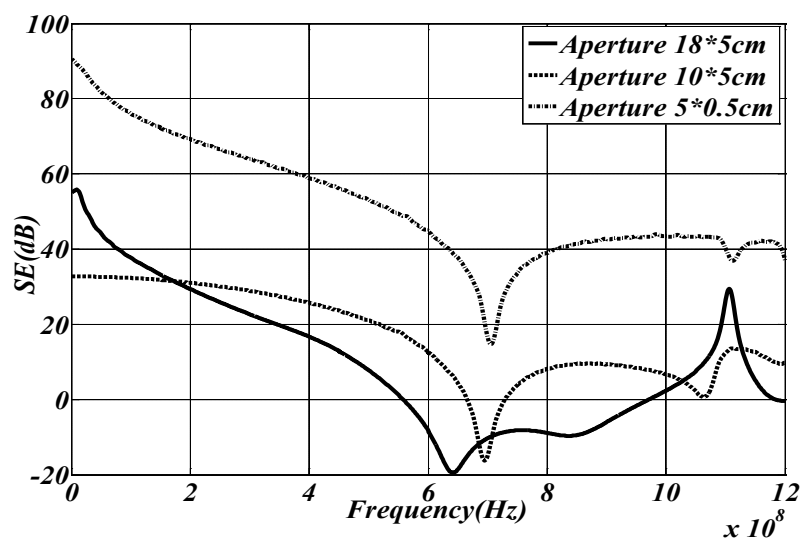

(c)

Figure 4. Different aperture sizes effect, a) $\left|S_{21}\right|$ comparison of unshielded and shielded MTL for decreasing $a^{\prime}$, b) $\left|S_{21}\right|$ comparison of unshielded and shielded MTL for decreasing $b^{\prime}$, c) SE Comparison of central point of a $30 \times 12 \times 30 \mathrm{~cm}$ empty enclosure with different apertures.

\subsection{Location of the MTL}

Two cases of shielded and unshielded MTL are considered.

\subsubsection{Unshielded MTL Case}

The distance between the antenna and MTL is chosen to be $\mathrm{d}=5.4,10.4$ and $15.4 \mathrm{~cm}$. Fig. 5 shows the resultant $\left|S_{21}\right|$ by CST and measurements. As observed, no significant susceptibility variation is produced by changing the position of the MTL. Here, again the measurements confirm the FIT results.

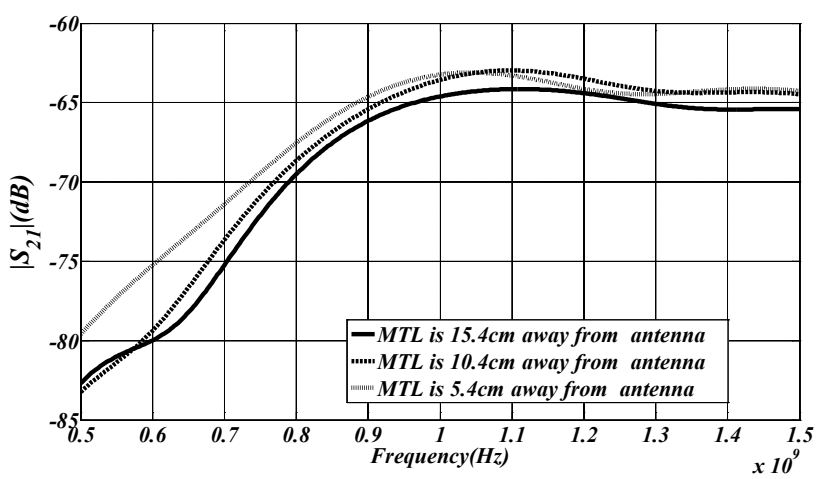

(a) 




(b)

Figure 5. $\left|S_{21}\right|$ Comparison of an unshielded MTL for different MTL locations, a) CST b) Measurements

\subsubsection{Shielded MTL Case}

The shield has a central $18 \times 5 \mathrm{~cm}$ aperture on its front wall. MTL is located at the central axis of the enclosure as in Fig. 1. Fig. 6.a-c compares CST results with measurements for different distances of $\mathrm{d}=5.4,10.4$ and $15.4 \mathrm{~cm}$ between MTL and the antenna. A very good agreement is observed in the results of Fig. 6 .

Fig. 7.a compares the effect of the considered distances on the susceptibility of the MTL. As illustrated, it cannot be decided exactly what distance gives better immunity for the MTL. For example, for frequencies up to $650 \mathrm{MHz}$, all the distances create approximately the same $\left|S_{21}\right|$. However, for higher frequencies up to around $950 \mathrm{MHz}$, the displacement of $5.4 \mathrm{~cm}$ improves $\left|S_{21}\right|$. For frequencies between $1.2 \mathrm{GHz}$ to $1.4 \mathrm{GHz}, \mathrm{d}=15.4 \mathrm{~cm}$ is a better option for the MTL location. Hence, to improve the susceptibility of PCBs inside an enclosure, an optimum location for PCBs according to the operating frequency, size of the enclosure and the apertures should be chosen.

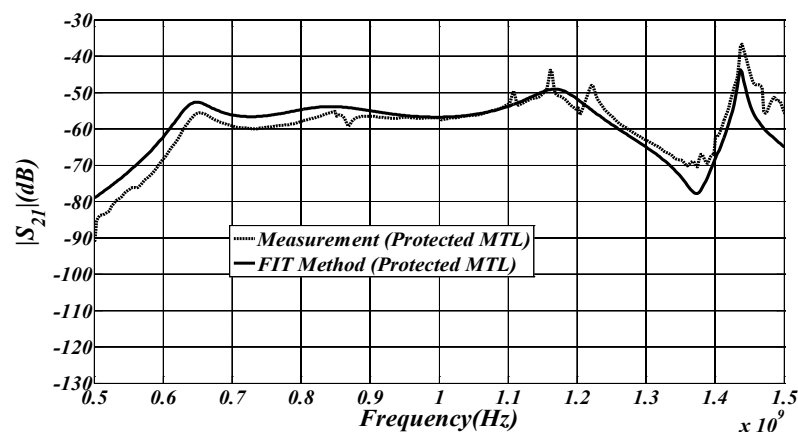

(a)

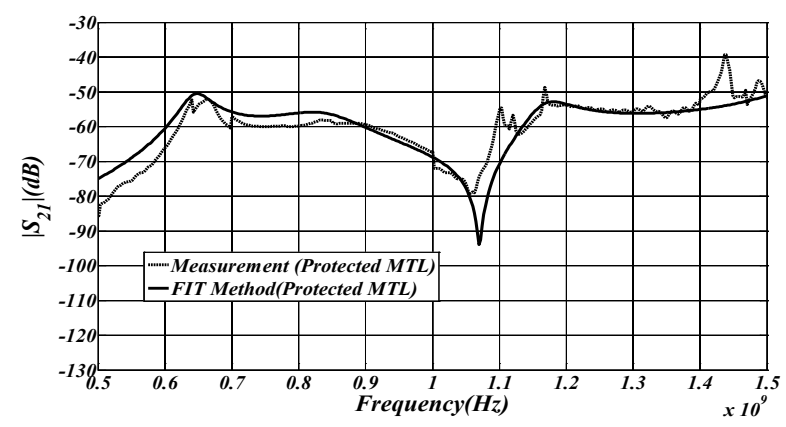

(b)

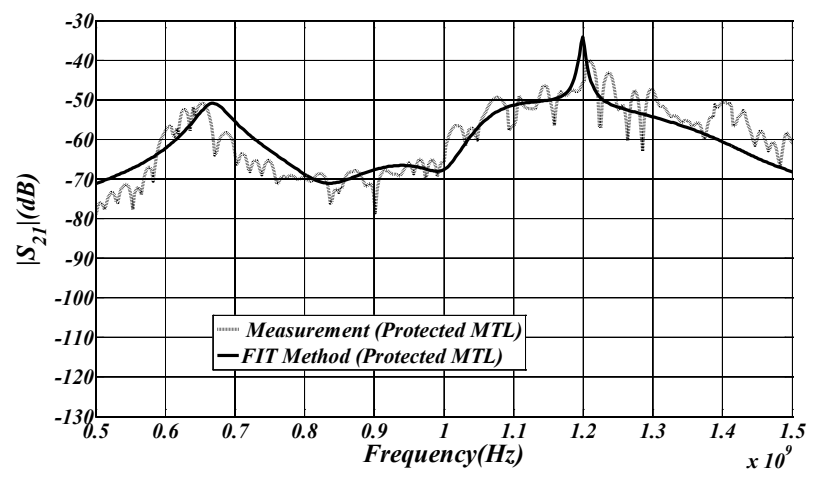

(c)

Figure 6. CST results and measurements for different MTL locations inside the same enclosure with $18 \times 5 \mathrm{~cm}$ aperture, a) $\mathrm{d}=15.4 \mathrm{~cm}, \mathrm{~b}) \mathrm{d}=10.4$ $\mathrm{cm}$ and, c) $\mathrm{d}=5.4 \mathrm{~cm}$



(a)

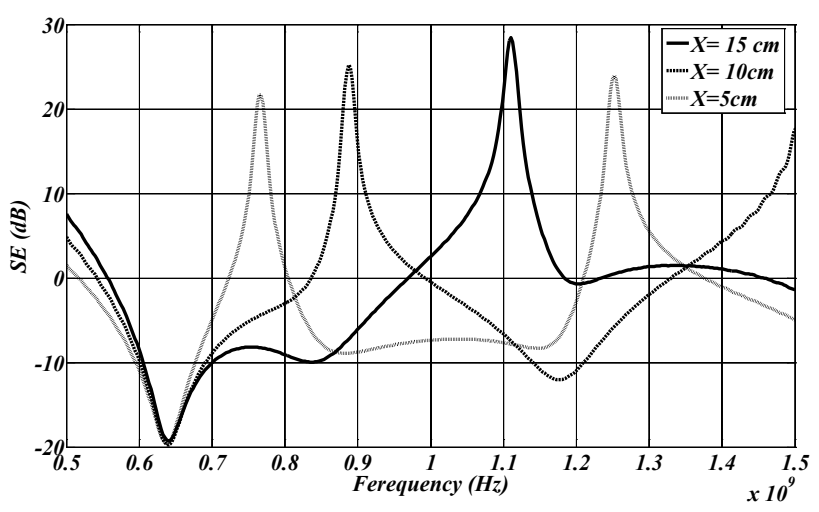

(b)

Figure 7. CST results for the same enclosure with a $18 \times 5 \mathrm{~cm}$ aperture a) susceptibility of MTL with $d=5.4, d=10.4$ and $15.4, b)$ SE of the empty enclosure at points $\mathrm{x}=5,10$ and 15 .

In addition, Fig. 7.b compares SE of the empty enclosure for the same points where MTL is placed in Fig. 7.a. It is observed that SE information can correctly predict the trend of susceptibility behavior versus frequency. As mentioned for Fig. 7.a, here in Fig. 7.b it is confirmed that changing the position of observed point inside the enclosure does not improve SE significantly; and it just makes shift in minima and maxima of the SE.

Fig. 8 shows the susceptibility of the MTL inside the same enclosure with aperture size of $5 \times 0.5 \mathrm{~cm}$. Similar to the large aperture case, the location of the MTL does not change the 
susceptibility of the MTL.

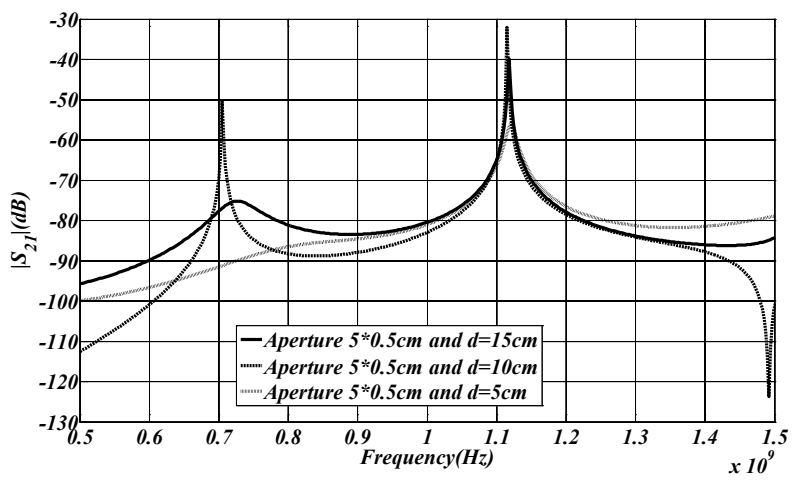

Figure 8. Susceptibility of the MTL with $d=5.4, d=10.4$ and $15.4 \mathrm{~cm}$ by CST for the enclosure with a $5 \times 0.5 \mathrm{~cm}$ aperture,.

\section{Conclusions}

In this paper, susceptibility of a simple MTL in the vicinity of an interfering antenna is studied. In this work, shielding enclosure with different aperture sizes is used to improve immunity of the MTL. It is shown that when the enclosure has a single central aperture and the antenna is placed centrally very close to the perforated wall, large size apertures degrade $\left|S_{21}\right|$ at larger bandwidth compared to the case where no shield is used. As the rectangular aperture length is reduced, $\left|S_{21}\right|$ decreases and gives better immunity for the MTL. However, $\left|S_{21}\right|$ demonstrates peaks at the enclosure resonant frequencies, and consequently the MTL is not immune at those frequencies. While reducing the length of the aperture improves the immunity of the MTL, decreasing the size of the aperture width does not affect the MTL susceptibility significantly, due to y-directed interfering antenna.

In addition, limited increase in distance between MTL and the antenna doesn't improve $\left|S_{21}\right|$, neither in shielded nor in unshielded case. And, an optimum distance between MTL and the antenna should be considered according to the frequency range, enclosure and aperture size. Furthermore in this paper, it is shown that the SE of an enclosure can be considered as a parameter to predict susceptibility of the shielded MTL against a very close interference.

\section{REFERENCES}

[1] R. P. Clayton, Introduction to Electromagnetic Compatibility, John Wiley \& Sons, New York, 2006.

[2] I. M. Mark and M. N. Edward, Testing For EMC Compliance Approaches and Techniques, John Wiley \& Sons, New York, 2004.

[3] M. Leone, Radiated Susceptibility on the Printed-Circuit-Board Level: Simulation and Measurement, IEEE Trans. On EMC (2005), 471-478.
[4] T. Namiki and K. Ito, Numerical simulation using ADI-FDTD method to estimate shielding effectiveness of thin conductive enclosures, IEEE Trans. on Microwave Theory and Techniques(2001), 1060-1066.

[5] M. Luo and K. Huang, Prediction of the Electromagnetic Field in Metallic Enclosures using Artificial Neural Networks, Progress In Electromagnetics Research, PIER (2011), 171-184.

[6] J. Chen and J. Wang, A Three-Dimensional Semi-Implicit FDTD Scheme for Calculation of Shielding Effectiveness of Enclosure With Thin Slots, IEEE Trans. On EMC (2007), 354-360.

[7] P. Dehkhoda, A. Tavakoli and R. Moini, An Efficient and Reliable Shielding Effectiveness Evaluation of a Rectangular Enclosure With Numerous Apertures, IEEE Trans. On EMC (2008), 208-212.

[8] M. Khorami, P. Dehkhoda, R. Moini and H.H.S. Sadeghi, Fast Shielding Effectiveness Calculation of Metallic Enclosures with Apertures Using a Multiresolution Method of Moments Technique, IEEE Trans. On EMC (2010), 230-235.

[9] S. Yenikaya and A. Akman, Hybrid MOM/FEM Modelling of Loaded Enclosure with Aperture in EMC Problems, International Journal of RF and Microwave Computer-Aided Engineering (2009), 204-210.

[10] B. Audone and M. Balma, Shielding Effectiveness of Slots in Rectangular Cavities, IEEE Trans. On EMC (1989), 102-106.

[11] D.W.P. Thomas, A.C. Denton, T. Konefal, T. Benson, C. Christopoulos, J. F. Dawson, A. Marvin, S.J. Porter, and P. Sewell, Model of the Electromagnetic Field Inside a Cuboidal Enclosure Populated with Conducting Planes or Printed Circuit Boards, IEEE Trans. On EMC (2001), 161-169 .

[12] K. Murano, T. Sanpei, F. Xiao, C. Wang, Y. Kami and J. L. Drewniak, Susceptibility Characterization of a Cavity With an Aperture by Using Slowly Rotating EM Fields: FDTD Analysis and Measurements, IEEE Trans. On EMC (2004), 169-177.

[13] C. Feng, and Z. Shen, A Hybrid FD-MoM Technique for Predicting Shielding Effectiveness of Metallic Enclosures with Apertures, IEEE Trans. On EMC (2005), 456-462.

[14] S. Atrous, D. Baudry, E. Gaboriaud, A. Louis, B. Mazari, and D. Blavette, Near-field Investigation of the Radiated Susceptibility of Printed Circuit Boards, 2008 IEEE International Symposium on Electromagnetic Compatibility- EMC Europe, Detroit, Sept. 2008, pp. 1-6.

[15] D. Jing and H. Guo, Study on Coupling Characteristics for EM Pulse into Slotted Shell of Loaded PCB, 2010 IEEE International Conference on Computer, Mechatronics, Control and Electronic Engineering (CMCE), Hong Guo, Aug. 2010, pp. 398-401.

[16] L. Tian-Hong, and M. J. Alexander, A Method to Minimize Emission Measurement Uncertainty of Electrically Large EUTs in GTEM Cells and FARs Above $1 \mathrm{GHz}$, IEEE Trans. On EMC ( 2006), 634-640.

[17] H. Tarhini, M. E. Haffar, C. Guiffaut, G. Andrieu, A. Reineix, B. Pecqueux, and J. C. Joly, Susceptibility of Printed Circuit Boards in Complex Electromagnetic 
Environment, 2008 IEEE International Symposium on Electromagnetic Compatibility- EMC Europe, Detroit, Sep. 2008, pp. 1-4.

[18] Y. Jiang, Z. Yan and T. Wang, A Behavioral Quantification Analytical Method of Electromagnetic Susceptibility at Board Level, 2010 IEEE International Symposium on Antennas Propagation and EM Theory, Guangzhou, Nov. -Dec. 2010, pp. 1051-1054.

[19] S. Abadpour, P. Dehkhoda, H. R. Karami and R. Moini, Aperture Size Effect on the Susceptibility of a PCB inside an
Enclosure, 2011 IEEE International Conference on Electromagnetics in Advanced Applications, Torin, Sep.2011, pp.741-744.

[20] www.CST.com.

[21] T. Weiland, A Discretization Method for the Solution of Maxwell's Equations for Six-Component Fields, Electronics and Communication (AEÜ) (1977), 116-124.

[22] J. G. Proakis and D. K. Manolakis, Digital Signal Processing, Principles, Algorithms and Applications, Prentice Hall, New York, 2006. 